\title{
Efecto de la aplicación de bioreguladores para el control de Moniliophthora roreri y Phytophthora palmivora en cacao CCN-51 (Theobroma cacao)
}

\author{
Effect of bioregulators application for the control of \\ Moniliophthora roreri and Phytophthora palmivora in cocoa \\ CCN-51 (Theobroma cacao)
}

\author{
Rodrigo Rodríguez Lozano*1 \\ ${ }^{1}$ Universidad Católica de Cuenca \\ *rrodriguez@ucacue.edu.ec
}

DOI: https://doi.org/10.26871/killkanatecnica.v4i2.280

\begin{abstract}
Resumen
El cacao ecuatoriano adquiere cada vez más importancia en los mercados internacionales. El desarrollo de técnicas sustentables es fundamental para productividad. El objetivo del presente estudio fue determinar el efecto de Bacthon y Tricho-D con dos periodos de aplicación para el control de Moniliophthora roreri y Phytophthora palmivora en cacao CCN-51. La investigación se desarrolló en la parroquia Pancho Negro, La Troncal, Cañar. Se analizaron tres métodos y dos periodos de aplicación más un testigo, con cuatro repeticiones. Se determino que el uso de Bacthon al suelo más Tricho-D a la planta ha superado en la mayor parte de las variables productivas, sanitarias y económicas del estudio. En cuanto a los periodos de aplicación evaluados, se obtuvo mejores resultados cuando las aplicaciones fueron realizadas con mayor frecuencia. Concluyendo que las aplicaciones con biorreguladores son más efectivas cuando se las realizan en menor lapso y directamente al suelo.
\end{abstract}

Palabras clave: Bacthon, Tricho-D, cacao CCN-51, Moniliophthora roreri, Phytophthora palmivora.

\begin{abstract}
Ecuadorian cocoa acquires more and more importance in international markets. The development of sustainable techniques is fundamental for its productivity. The objective of this study was to determine the effect of two application periods of Bacthon and Tricho-D for the control of Moniliophthora roreri and Phytophthora palmivora. The research was developed in a cocoa CCN-51 crop in Pancho Negro parish, La Troncal, Cañar. Three methods combined with two periods of application were used, plus a control treatment. All treatments have four repetitions. The study determined that the use of Bacthon and Tricho-D applied directly to plant soil, had better results in most of the productive, sanitary and economic variables. Better results were obtained when applications were made more frequently. Concluding that applications with bioregulators are more effective when they are carried out in less periods of time and directly to the ground.
\end{abstract}

Keywords: Bacthon, Tricho-D, Moniliophthora roreri, Phytophthora palmivora, cacao CCN-51.

\section{INTRODUCCIÓN}

El cacao (Theobroma cacao L), originario del alto Amazonas de América del Sur, se divide en dos grupos según sus características fenotípicas y genotípicas: el Criollo o de aroma es de color rojo-amarillo al estar maduro, su origen es centro y sudamericano, teniendo una alta susceptibilidad a las principales enfermedades [1], el Forastero que es la variedad más común y de mayor rendimiento, aunque su aroma es de menor intensidad [2] y el cruzamiento entre los dos, denominado Cacao Trinitario [3] que es el de mejor genotipos desarrollando una mejor resistencia a plagas y un buen rendimiento [4].

En Ecuador existe una alta diversidad genética de esta especie [5] donde la actividad cacaotera involucra 500000 hectáreas; y es cultivado en 16 provincias de la costa, amazonia y en las estribaciones de la cordillera [6] siendo un cultivo de gran potencial económico [7] y constituye uno de los principales rubros de exportación [8] respondiendo a una demanda creciente [9]. Entre las principales enfermedades que causan grandes pérdidas en la producción 
de cacao están: la Moniliasis (Moniliophthora roreri) que ataca las mazorcas en cualquier estado de desarrollo [5] produciendo pérdidas económicas de gran importancia y la mazorca negra (Phytophthora palmivora), que ataca a la mazorca, los brotes tiernos, el tronco, los cojinetes florales y las raíces [10]. Siendo su impacto económico leve, dado que la especie de Phytophthora palmivora local es diferente a la de otras regiones [5].

La utilización de agentes biológicos como Trichoderma ha despertado intereses debido a su efecto antagónico contra un amplio rango de hongos fitopatógenos, como Monilla [11] ya que no son productos tóxicos y no contaminan el ambiente.

El propósito de este estudio fue evaluar el efecto productivo, sanitario y económico de dos biorreguladores en un cultivo de cacao, aplicándolos en diferentes tiempos y métodos. Las variables productivas y sanitarias fueron el porcentaje de mazorcas sanas, porcentaje de mazorcas cherelles o pasmadas, porcentaje de mazorcas infectadas por Monilia antes de la cosecha, porcentaje de mazorcas infectadas por Phytophthora, porcentaje del área infectada por Monilia en las mazorcas después de la cosecha, longitud de mazorcas, diámetro de mazorcas, peso de mazorcas y peso de almendras secas.

\section{A. CLON CCN-51}

El clon CCN-51, es una variedad de fácil manejo, precoz, de alta productividad, resistente a escoba bruja, con mazorca y semillas grandes [12], tiene las siguientes características: La raíz pivotante que puede crecer entre $1.20 \mathrm{~m}$ a $1.50 \mathrm{~m}$ llegando en suelo sueltos hasta $2 \mathrm{~m}$. Las raíces secundarias se hallan a una profundidad de 25 a $30 \mathrm{~cm}$ del suelo alrededor del árbol [13]. El tronco de color castaño varía su ángulo de crecimiento, pudiendo medir hasta $4 \mathrm{~m}$ de altura. Las flores hermafroditas y pentámeras [14] tienen cáliz de color rosa con segmentos puntiagudos, [15] la corola es de color blanco, amarillo o rosa y los pétalos son largos [16]. Aparecen insertadas sobre el tronco en forma de racimo o en ramificaciones viejas [17]. Las hojas son simples y de peciolo corto. Su crecimiento varía dependiendo la intensidad de la luz. Las hojas tiernas son de color verde pálido a violeta y a medida que van toman un color verde oscuro. La longitud de las hojas varía de 30 a $50 \mathrm{~cm}$ viéndose afectado su crecimiento por la intensidad de la luz a la que están expuestas[18]. Los frutos son bayas indehiscentes de forma variada y color morado de tiernas y rojizas o amarillas al madurar. Tienen cinco lóculos en su interior donde se encuentran las semillas [5] cuya cantidad depende del número de óvulos en cada ovario. Las mazorcas están unidas al tronco por un pedúnculo grueso y el tiempo desde la fecundación hasta la madurez es alrededor de 180 días [19]. Su producción tiene un promedio de 45 granos x mazorca $(1.5 \mathrm{~g})$ tiene una dimensión de 20 a $30 \mathrm{~mm}$. x 10 a $17 \mathrm{~mm}$; con un porcentaje del peso de almendra de 84.8 [20], estas semillas están unidas a la placenta por la placenta [5] de color blanco a violeta [17].

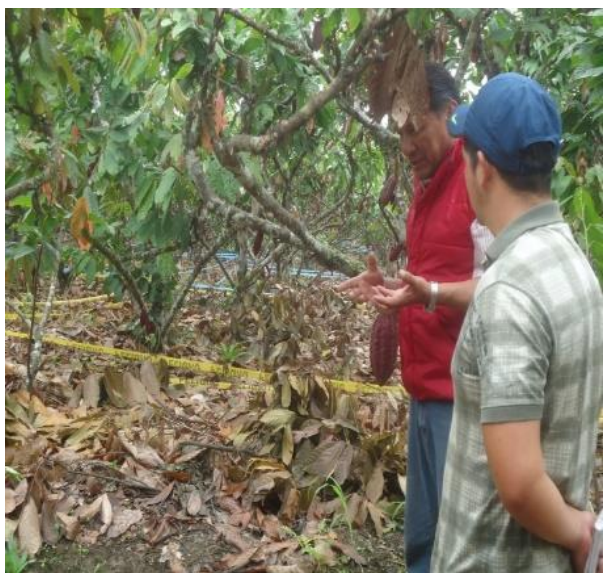

Fig. 1: Visita técnica de campo

Fuente: Autor

\section{Requerimientos del cultivo}

Necesita suelos con una ligera inclinación, sueltos y profundos, ricos en materia orgánica, con $\mathrm{pH}$ entre 6,0 y 7,0 [21]. La precipitación óptima es de 1600 a 2500mm en zonas cálidas y 1200 a $1500 \mathrm{~mm}$ en valles altos. Precipitaciones mayores a los $3800 \mathrm{~mm}$ causan enfermedades y saturan los suelos; mientras que las inferiores a los 1600 requieren de riego artificial [22]. La temperatura está relacionada con el desarrollo, floración y fructificación. El margen de temperatura optimo es de 18 y $32^{\circ} \mathrm{C}$ siendo el ideal los 24 a $28^{\circ} \mathrm{C}$ [23].

Además, es necesario considerar la velocidad de viento ya que este provoca defoliaciones muy fuertes [24] Se desarrolla a altitudes inferiores de $800 \mathrm{msnm}$ y el que se desarrolla a altitudes mayores a los $1000 \mathrm{msnm}$ es ampliamente reconocido [25]. La intensidad de la luz es importante ya que las plantas son muy susceptibles a la acción directa de los rayos solares [26]. El cacao se desarrolla bien con una humedad relativa del $80 \%$ [27]. Las condiciones ambientales desfavorables provocan enfermedades [28].

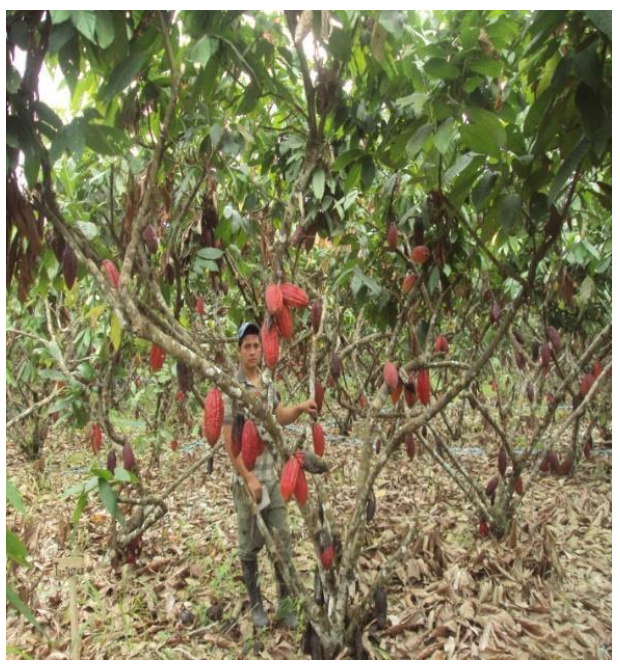

Fig. 2: Plantación de cacao Fuente: Autor 


\section{B. Plagas y Enfermedades}

En el Ecuador las principales plagas que causan daño en el cultivo de cacao y se vuelven un problema económico son las siguientes: Hormigas arrieras; Atta sp. [5]; Pulgones, Aphis sp [29]; Chinches de cacao, Monalonium sp [30]; El barrenador del tronco, Xyleborus sp [29]; Trips, [31].

En el Ecuador las enfermedades del cacao causan más pérdidas para los agricultores que los insectos, causando hasta el $80 \%$ de pérdidas en la producción, entre ellas: Escoba de bruja, Crinipellis perniciosa [32, 33]; Mal de machete Ceratocystis fimbriata [34].

\section{Mazorca Negra (Phytophthora palmivora)}

La Phytophthora palmivora llamada mazorca negra [40] que es una enfermedad que causa pérdidas anuales superiores al $30 \%$ y dependiendo de las condiciones ambientales pueden alcanzar un $60 \%$ [35]; atacando con más severidad en la época invernal, diseminándose en el suelo [36] afectando diferentes partes del árbol de cacao entre ella las mazorcas bajeras [37]. Sus síntomas se presentan como manchas de color café oscuro, con bordes parejos y cubren todo el fruto que se pone blandos [38]. El hongo se reproduce de forma asexual con la formación de clamidiosporas [39] y esporangios [40] que contienen las zoosporas y de forma sexual mediante la formación de oósporas. Las esporas producen el micelio y permite al hongo propagarse y sobrevivir [41].

\section{Monilia (Moniliophthora roreri)}

La Moniliophthora roreri un hongo de la Familia de las Marasmiaceae [42] presenta entre otros nombres comunes como: Moniliasis del cacao, Pudrición del fruto, Pudrición acuosa del fruto o Pasmo [43]. Esta enfermedad originaria del noreste de Colombia es una de las enfermedades altamente destructiva. Esta enfermedad afecta únicamente al fruto, afectando desde las etapas iniciales del mismo [44] hasta la cosecha, donde aparecen frutos de apariencia sana pero con su interior dañado [45]. Externamente se puede reconocer puntos circulares aceitosos muy pequeños donde se desarrolla el micelio, seis a diez posteriores a la infección [46]. Una vez que el patógeno penetra en el fruto este se desarrolla intracelularmente manifestando los signos de la enfermedad mucho tiempo después [47] dependiendo de las condiciones ambientales del cultivo como humedades relativas superiores a $85 \%$ o temperaturas de más de $25^{\circ} \mathrm{C}$ [8] llegan a dañar hasta el $80 \%$ del fruto [5].

\section{Prevención y control}

Para prevenir la enfermedad se debe retirar los frutos que presenten los signos de las enfermedades [10] evitando la bioacumulación de los residuos de la cosecha. Cuando las enfermedades atacan otras partes de la planta se debe retirar la parte infectada realizándole una poda y utilizando fungicidas preventivos como $\mathrm{CuO}_{2}, \mathrm{Cu}_{2}(\mathrm{OH})_{3} \mathrm{Cl}, \mathrm{CuSO}_{4}$ pentahidratado [48] ó el uso de en la aplicación de Clorothalonil [44]. El control cultural debe enfocarse en evitar la entrada del patógeno al cultivo, impidiendo que encuentre las condiciones favorables de infección y diseminación [5]. Entre las practicas más comunes esta la poda racional para reducir sombra y separar ramas secas que puedan estar infectadas [49], control de malezas [50], eliminación de mazorcas enfermas [45], manejo de residuos de cosecha [10] control genético [47] con el uso de cultivares resistentes [51] y el control químico [52]. Sin embargo, este último resulta muy costosos y en caso ce Moniliasis poco efectivo [53].

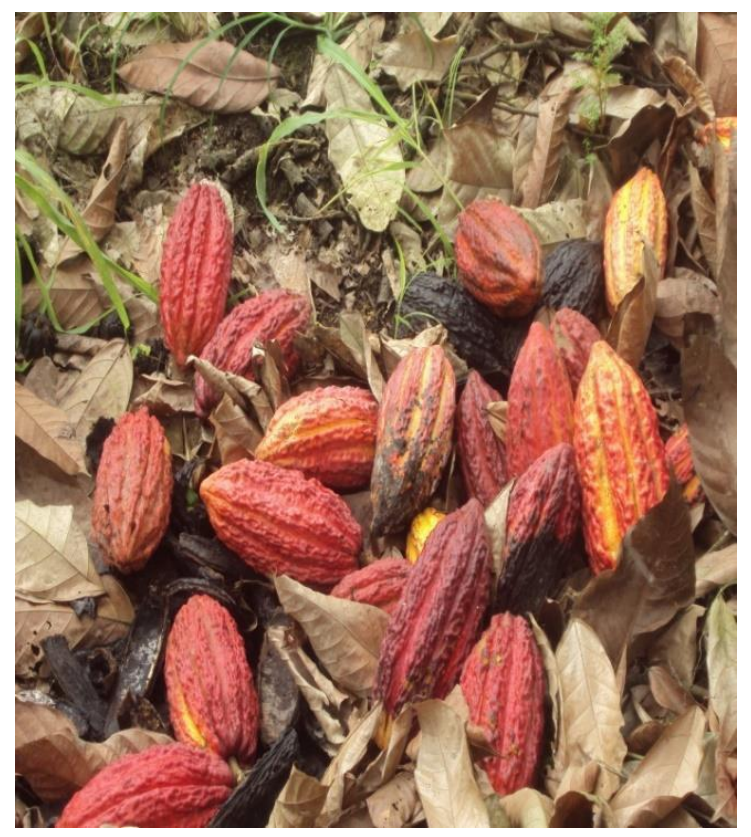

Fig. 3: Mazorcas Cosechadas Fuente: Autor

\section{CONTROL BIOLÓGICO}

Se basa en la implementación de microorganismos antagonista [54] como herramienta de reducción del inoculo de un patógeno [55]. El género Trichoderma es muy utilizado en el control biológico de hongos que se desarrollan en el suelo, el follaje [56] e inhibe el desarrollo de otros hongos de las flores y los frutos como los del género Monilia [46]. Trichoderma se encuentra en la hojarasca por lo que controla las mazorcas infectadas que están bajo la misma [57].

\section{A. Biocontroladores Comerciales}

BACTHON SC es un Inoculante Biológico formulado con microorganismos beneficos del suelo, (Azospirillum brasilense, Azotobacter chroococcum, Lactobacillus acidophillus, Saccharomyces cerevisae) que mejora el suelo agrícola al limpiarlo de las toxinas y agroquímicos, transformando los residuos de los cultivos hasta convertirlos en una fracción orgánica del suelo [58]. Su aplicación se la realiza al suelo y es compatible con la mayoría de los agroquímicos. 
TRICHO-D formulado con nutrientes y esporas en latencia del hongo Trichoderma harzianum es un acondicionador de suelo que actúa en la biorregulación de los principales fitopatógenos que enferman los cultivos agrícolas [58]. $\mathrm{Su}$ aplicación se la realiza en aspersión dirigida al suelo húmedo. Este producto presenta una ligera toxicidad para el operario.

\section{Metodología}

La investigación se realizó en 504 plantas de cacao CCN51 de 15 años de edad, sembradas a $3 \times 3 \mathrm{~m}$, cultivadas en el cantón La Troncal (Cañar) a 200msnm; con una temperatura media de $24.6^{\circ} \mathrm{C}\left(+/-4^{\circ} \mathrm{C}\right)$; con 1400 a 2000 mm de precipitación y humedad relativa del $88 \%$. [59], a las que se les aplico dos biorreguladores (Bacthon SC y Tricho-D). Se utilizaron siete tratamientos combinando las prácticas culturales (períodos) y la aplicación de biorreguladores (métodos) según se muestra en la Tabla I.

TABLA I

\begin{tabular}{lll}
\hline Nomenclatura & Método (por planta) & Periodo \\
\hline M1.P1 & Bacthon 7.5 $\mathrm{cm}^{3}+$ Tricho-D al suelo1.5g & Dos meses \\
M1.P2 & Bacthon $7.5 \mathrm{~cm}^{3}+$ Tricho-D al suelo1.5g & Tres meses \\
M2.P1 & $\begin{array}{l}\text { Bacthon } 7.5 \mathrm{~cm}^{3} \text { al suelo + Tricho-D a la } \\
\text { planta 1.5g }\end{array}$ & Dos meses \\
M2.P2 & $\begin{array}{l}\text { Bacthon } 7.5 \mathrm{~cm}^{3} \text { al suelo + Tricho-D a la } \\
\text { planta 1.5g }\end{array}$ & Tres meses \\
M3.P1 & Tricho-D a la planta 1.5g & Dos meses \\
M3.P2 & Tricho-D a la planta 1.5g & Tres meses \\
TESTIGO & - & - \\
\hline
\end{tabular}

Fuente: Autor

Se realizó una poda sanitaria un mes antes de la aplicación. El control de malezas se realizó mediante la fumigación química cada cuatro meses. Se realizaron riegos específicos cada 15 días. La cosecha se realizó en el momento que los frutos mostraron madurez fisiológica; realizándose dos cosechas en el transcurso de la investigación. Para finalmente realizar un análisis económico en base a los costos de producción. Se utilizó el D.B.C:A. en arreglo factorial M (3) x P (2) + Ts.

\section{REsultados}

Se estudiaron nueve variables de interés comercial que cuantificaron valores sanitarios y productivos. Al realizar un análisis de las correlaciones de Pearson entre las variables existentes en el estudio se confirmó la alta relación existente ( $p$ 0.05) entre el Peso Total de la Mazorca con el Peso de Cacao Seco $\left(0.70^{* *}\right)$ y una relación altamente significativa entre Longitud y Diámetro $\left(0.76^{* *}\right)$. Además de una relación negativa $(-0.99 * *)$ entre la presencia de mazorcas sanas con las que presentan problemas sanitarios. De las demás correlaciones hechas ( $\mathrm{p}$ 0.05) entre las variables, destaca la existente entre el Peso Total de la Mazorca y el Porcentaje de Phytoptora $(-0.38 *)$; explicando de este modo el efecto perjudicial del hongo en el Peso Final de la Mazorca.

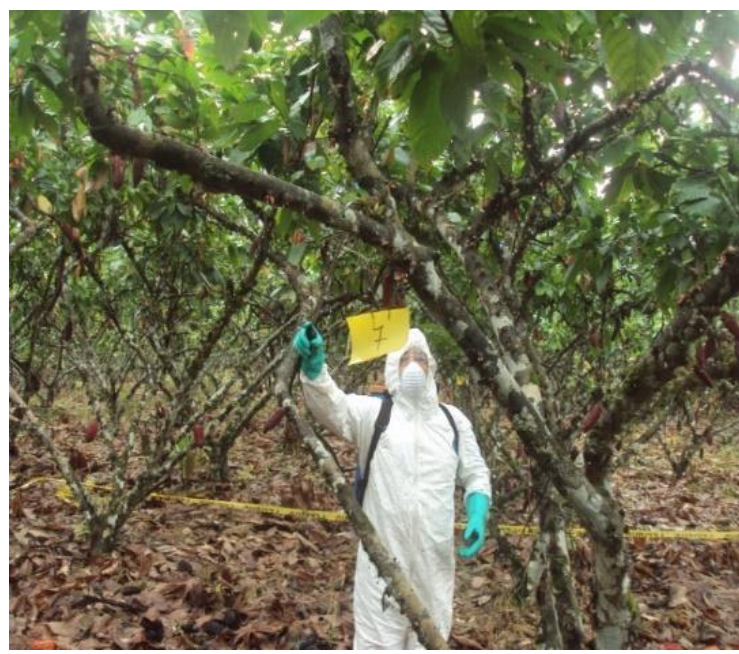

(a)

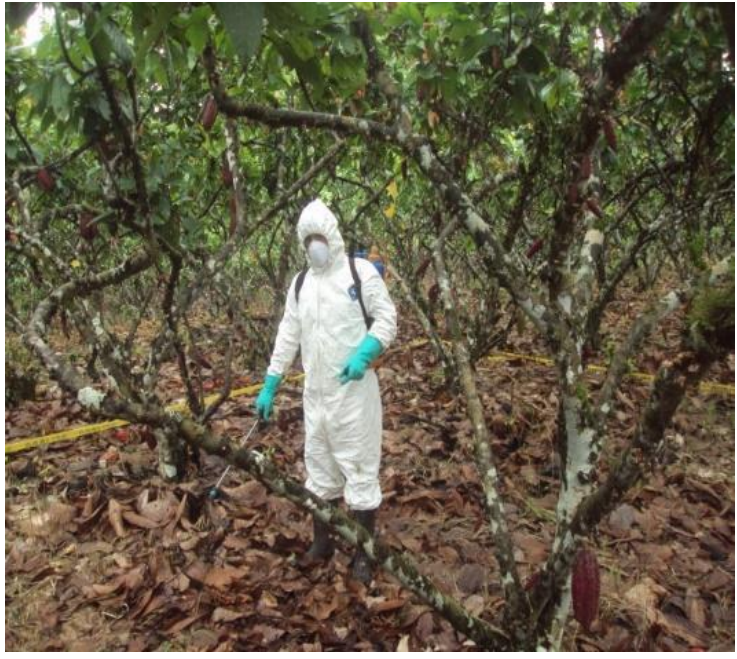

(b)

Fig. 4: Aplicación de bioreguladores a la planta y al suelo en el campo de estudio

\section{A. Factores Sanitarios}

Al segundo mes después de la aplicación de los biorreguladores se contó el número de todas las mazorcas de cada unidad experimental, sacando el porcentaje de mazorcas pasmadas o cherelles; los mismos se pueden observar en la Tabla II, sin que existan diferencias significativas entre tratamientos, en el análisis de varianza del porcentaje de mazorcas cherelles o pasmadas al segundo mes de aplicación. 
TABLA II: Porcentaje de mazorcas cherelles al segundo mes de la aplicación

\begin{tabular}{|c|c|c|c|c|c|c|}
\hline \multicolumn{7}{|c|}{ Repeticiones } \\
\hline TRATAMIENTO & I & II & III & IV & SUMA & PROMEDIO \\
\hline M1P2 & 5,7 & 5,8 & 4,4 & 6,4 & 22,3 & 5,575 \\
\hline M2P2 & 4,3 & 4 & 5,8 & 6,5 & 20,6 & 5,15 \\
\hline M3P1 & 5,4 & 4,4 & 5,8 & 5,1 & 20,7 & 5,175 \\
\hline M3P2 & 4,8 & 6,7 & 3,7 & 6,3 & 21,5 & 5,375 \\
\hline Suma total & 37,3 & 37,6 & 36,5 & 42,6 & 154 & 38,5 \\
\hline Promedio & & & & & & 5,5 \\
\hline
\end{tabular}

Fuente: Autor

El porcentaje de mazorcas sanas con respecto a los Tratamientos se representan en el Gráfico 5; donde las aplicaciones hechas cada dos meses directamente a la planta Tricho$\mathrm{D}$ a la planta $1.5 \mathrm{~g},(54.68 \%)$ presentan un mayor porcentaje de mazorcas sanas, junto a las aplicaciones hechas igual cada dos meses, pero con Bacthon $7.5 \mathrm{~cm} 3$ al suelo + Tricho-D a la planta $1.5 \mathrm{~g}(47.78 \%)$. Los valores inferiores fueron los del método Bacthon $7.5 \mathrm{~cm} 3+$ Tricho-D al suelo $1.5 \mathrm{~g}$, con periodos de aplicación de dos $(38.10 \%)$ y tres $(39.00 \%)$ meses.

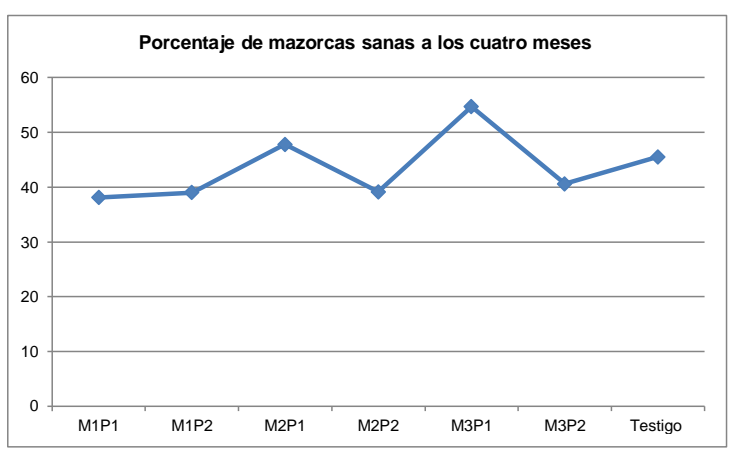

Fig. 5: Porcentaje de mazorcas sanas a los cuatro meses Fuente: Autor

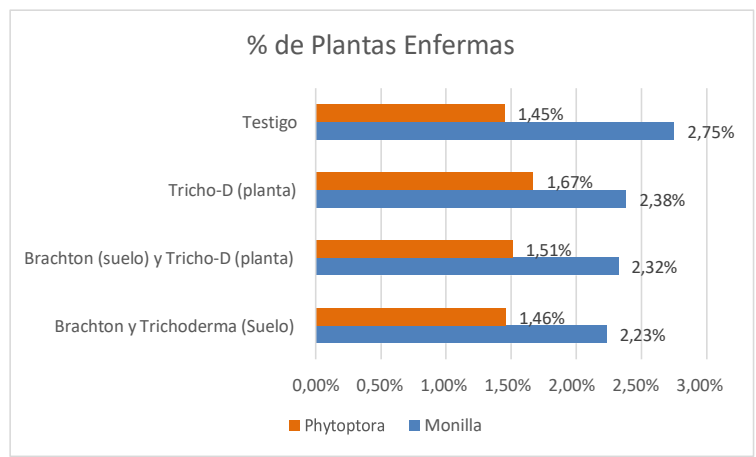

Fig. 6: Porcentaje de Plantas enfermas en relación con el método de aplicación

Fuente: Autor

El Gráfico 6, demuestra el porcentaje de plantas enfermas con Monillia y Phytophtora, por Método, donde los valores de mayor presencia de Monillia tiene el testigo
(2.75\%); mientras que Phytophtora, afecta en mayor porcentaje a las plantas tratadas con Tricho D (1.67\%). Con respecto a los periodos de aplicación el Gráfico 7 , indica que el testigo es el que tiene más área infectada $(76.75 \%)$

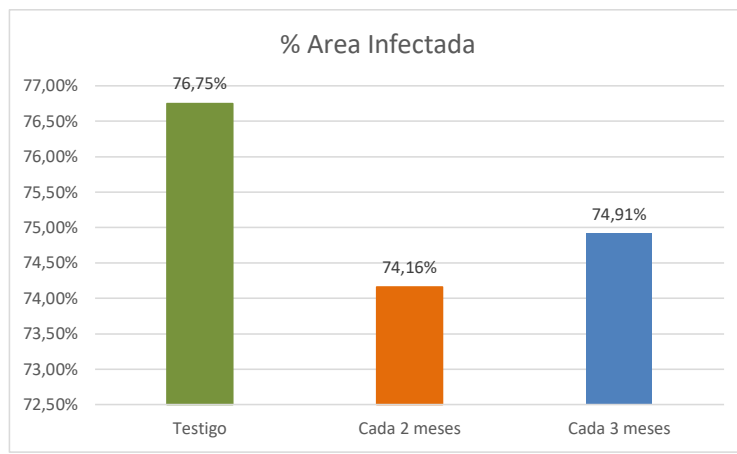

Fig. 7: Área Infectada por Monilla con respecto al Periodo Fuente: Autor

\section{B. Factores productivos}

Al comparar los dos periodos de aplicación en forma individual con respecto al peso de la mazorca seca, se pudo diferenciar que los pesos de la mazorca seca en las aplicaciones cada dos meses $(2.45 \mathrm{~kg}$.)a son estadísticamente superiores a las aplicaciones cada tres meses $(1.81 \mathrm{~kg}$.)b y Testigo (1.82kg.)b, valor referenciado en el Graficos 8. La variación de los rendimientos en grano seco por tratamientos se expresa en la Tabla III.

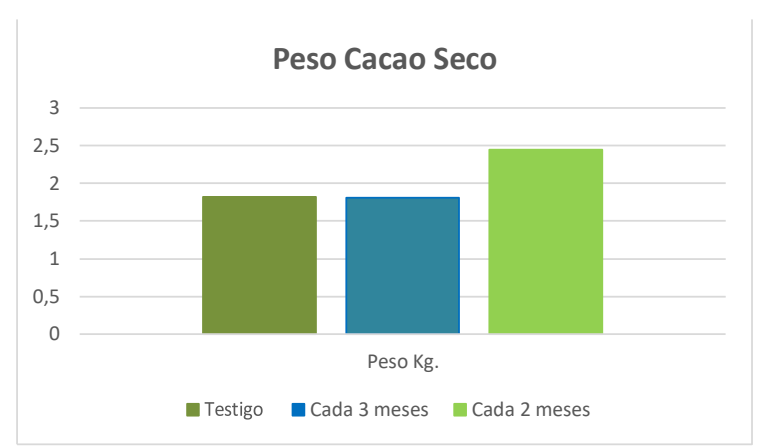

Fig. 8: Área Infectada por Monilla con respecto al Periodo Fuente: Autor 
TABLA III: Rendimiento de Distintos Periodos (Kg/mazorca) de Cacao Seco

\begin{tabular}{ll}
\hline Tratamientos & Pesos \\
\hline M1.P1 & $2.250 \mathrm{~kg} \cdot{ }^{b, c}$ \\
M1.P2 & $2.025 \mathrm{~kg} \cdot{ }^{b, c}$ \\
M2.P1 & $2.600 \mathrm{~kg} \cdot{ }^{a}$ \\
M2.P2 & $1.625 \mathrm{~kg} \cdot{ }^{c}$ \\
M3.P1 & $2.525 \mathrm{~kg} \cdot{ }^{b}$ \\
M3.P2 & $1.800 \mathrm{~kg} \cdot{ }^{b, c}$ \\
TESTIGO & $1.825 \mathrm{~kg} \cdot{ }^{b, c}$ \\
\hline
\end{tabular}

Fuente: Autor

El efecto de diferentes periodos y metodologías sobre las dimensiones de la mazorca de cacao es mínimo, según se puede apreciar en el Gráfico 9.

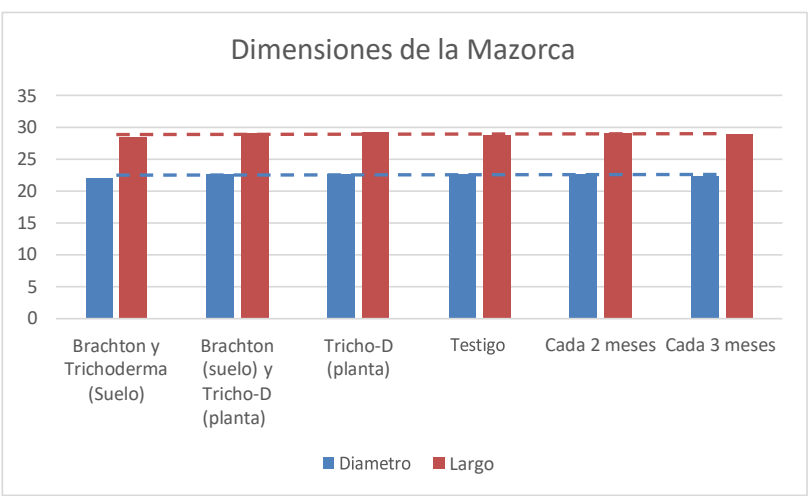

Fig. 9: Relación de Dimensión de mazorcas después de la cosecha

Fuente: Autor

Las aplicaciones de biorreguladores cada dos meses provocan un mayor beneficio económico neto sobre los demás tratamientos, llegando a superar en más del $20 \%$ del beneficio sobre las prácticas culturales tradicionales, especialmente cuando la aplicación del Trichoderma es Foliar. Cuando las aplicaciones de los biorreguladores superan los 2 meses los beneficios económicos netos son menores con respecto a los del Testigo, según se representa en el Gráfico 10 .

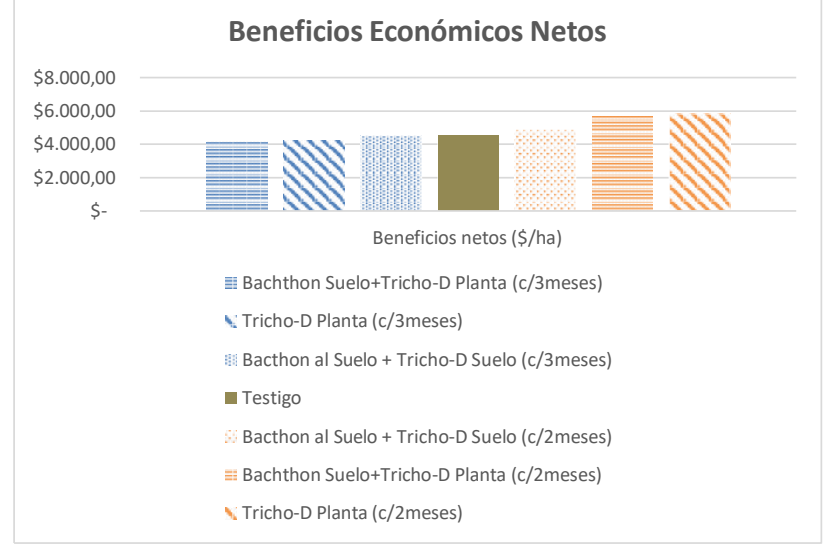

Fig. 10: Beneficios Económicos Netos Fuente: Autor

\section{DISCUSIÓN}

La producción de cacao se ve limitada por la presencia de enfermedades que causan grandes pérdidas que van desde el $20 \%$ hasta un $80 \%$ de la producción según las condiciones ambientales, el manejo del cultivo y variedad de mismo [11]. El comparar biorreguladores en una variedad híbrida, como el clon CCN-51 sirve para cuantificar el impacto productivo, sanitario y económico de los mismos en el cultivo; porque si bien esta variedad ofrece cierta resistencia a enfermedades simultáneamente existen reportadas muchas pérdidas en la producción debido a las enfermedades [12] debido a la variedad de condiciones ambientales y tipos de manejo. La poca variabilidad de los resultados entre repeticiones, nos indica que a pesar de que existan cambios climatológicos los resultados guardan robustez, evitando la incidencia del ambiente que indica Drenth, A (2013) [28].

Este estudio se lo realizo en la variedad de cacao hibrido de mayor demanda por el productor, bajo un manejo técnico-tradicional y en una zona altamente productiva, lo que ayuda a extrapolar los resultados a un mayor número de casos donde Phytophtora y Moniliophthora roreri ataca.

En este estudio el mayor porcentaje de frutos dañados se presenta por Moniliasis debido a que en el cultivo de cacao Moniliophthora roreri afecta únicamente al fruto [44]. Phytophtora puede atacar diferentes partes del árbol de cacao como cojinetes florales, chupones, brotes, hojas, ramas, tronco [37], lo que incide en un aumento de mazorcas cherelles o pasmadas. Las aplicaciones directas al suelo al suelo ayudan a controlar los estadios iniciales del hongo, corroborando las afirmaciones de la CORPEI (2013) que indican que Phytophtora se reproducen en las primeras capas del suelo.

Trichoderma prospera en el suelo del agroecosistema del cacao como un antagonista natural de Moniliophthora roreri [57]. La aplicación de biorreguladores, tienen que ser acompañadas de condiciones ambientales que favorezcan el desarrollo del producto biorregulador [58] razón por la que las aplicaciones que se realizaron directamente al suelo en este estudio fueron las que obtuvieron mejores resultados. 
En cultivos de ciclo corto se debe aplicar los biorreguladores al suelo húmedo, en capacidad de campo al momento de la siembra y repetir su aplicación a los 30 días [58], durante este estudio se realizó aplicaciones en rangos de tiempo mayores, sin embrago, los mejores resultados se obtuvieron cuando esta frecuencia fue más corta, tal como indican las recomendaciones del producto. Bacthon es un biorregulador que promueve el desarrollo de las plantas [58], por lo que su efecto en un cultivo ya establecido necesitaría de más tiempo de estudio, mientras que el efecto de Tricho-D es fácilmente cuantificable como sucede en esta investigación, debido a que se reproduce en la hojarasca.

\section{CONCLUSIONES}

El estudio demostró que las aplicaciones frecuentes de biorreguladores (P1=cada 2 meses), en particular de Trichodema foliar (Método 2 y 3 ) producen mejores promedios sin llegar a ser significativos en todas las variables. Al comparar específicamente las frecuencias de aplicación con los pesos del grano seco las aplicaciones bimensuales resultan superiores y en los beneficios económicos los tratamientos foliares y más frecuentes demuestran tener más beneficios.

La aplicación del tratamiento tres (M2.P1) Bacthon al suelo más Tricho-D a la planta aplicado cada dos meses presentó mejores resultados para porcentaje de mazorcas sanas, frutos cherelles, infectadas por Phytophthora y peso de las almendras secas en comparación al resto de tratamientos

En esta investigación la aplicación de Bacthon y Tricho$\mathrm{D}$ cada dos meses se vio reflejada en el incremento de peso en mazorcas y almendras secas con relación al testigo.

La mejor producción de cacao se observó en los tratamientos tres (M2.P1) y cinco (M3.P1) con un promedio de $2,6 \mathrm{~kg}$ de almendra seca por planta, en base al testigo con $1,8 \mathrm{~kg}$.

De los resultados obtenidos en la investigación se recomienda probar diferentes dosis de biorreguladores en función a las distintas condiciones climáticas, labores culturales, edad del cultivo y tercio de producción.

\section{REFERENCIAS}

[1] AYESTA, E. Caracterización morfológica de Theobroma caca L. Managua: Universidad Nacional Agraria. 2009

[2] SORIANO, G. Conoce los tipos de cacao que hay. Alicante: Chocolates Valor. 2012

[3] BATISTA, L. El cultivo de cacao. Santo Domingo: Teófilo Suriel. 2009

[4] INTA. Guía tecnológica del cultivo de cacao. IV. 2010

[5] AYALA, F. Manejo integrado de moniliasis en el cultivo de cacao. Guayaquil: Escuela Superior Politecnica del Litoral. 2008

[6] PICO, J. Guía del manejo integrado de enfermedades del cacao en la Amazonia. Orellana: INIAP. 2012

[7] ESTRADA, W. Guía técnica del cultivo de cacao. San Salvador: CIETTA. 2011
[8] ROBLES, B. Validación de biopesticidas para el control de moniliasis en cacao. Santo Domingo: Escuela Politecnica del Ejercito. 2008

[9] NADURILlE, E. Cacao: Cadena de valor de Costa Rica. Costa Rica: IICA- CATIE. 2010

[10] ASTORGA, C. Enfermedades del cacao y su control. Proyecto Cacao. 2008

[11] OSORIO, R. Estudio del efecto de Trichoderma harzianum en el control de monilia. Quito: Escuela Politecnica Nacional. 2010

[12] CEDEÑO, S. La revolucion del cacao CCN51 en el Ecuador. Naranjal: Industrial Agricola Cañas. 2011

[13] GUALPA, F. Proyecto de riego por goteo y microaspersión para el cultivo de cacao. Santo Domingo: ESPE. 2014

[14] GARCÍA, L. Estudio de caracterización del potencial genético del cacao. Lima: CONSULTING S.A.C. 2009

[15] CÓRDOBA, C. Manejo agroecológico del cacao . Costa Rica : FUNDESYRAM. 2014

[16] GUAMAN, c. Estudio de factibilidad para el cultivo de cacao CCN51. Santo Domingo: Escuela Politecnica Nacional. 2009

[17] CARMONA, J. Estudio del cacao. Quito: Universidad Internaciona Del Ecuador. 2012

[18] EGUINGUEREN, A. Estudio del cacao y propuesta gastronómica de autor. Quito: Universidad Internacional Del Ecuador. 2012

[19] PAREDES, N. Manual de cultivo de cacao. Quito: Activa Diseño Editorial. 2009

[20] BARONA, V. slideshare.net. (Turismo Negocios) Recuperado el 25 de julio, de slideshare: www.es.slideshare.com 2009

[21] TORRES, L. Manual de producción de cacao fino de aroma. Cuenca: Universidad De Cuenca. 2012

[22] MOSCOL, M. Manual de manejo tecnico del cacao. Lima: Ministerio de Agricultura. 2012

[23] ROJAS, F. Guia ambienal para el cultivo del cacao. Bogotá: MinAgricultura. 2013

[24] YÁNEZ, C. Plan de exportacion de pasta de cacao hacia el país de Japón. Riobamba: Escuela Superior Politécnica de Chimborazo. 2011

[25] SÁNCHEZ, E. Guía ambiental para el cultivo de cacao. Bogotá: FEDECACAO. 2009

[26] RAMÍREZ, M. Programa estratégico para el desarrollo rural sustentable. Tabasco: INIFAP. 2011

[27] ROMERO, K. Propuesta de un diseño agroforestal con cacao. Cartago: Tecnológico de Costa Rica. 2014

[28] DRENTH, A. Phytophthora: la destructora de plantas. XXXIV(49). 2013

[29] PÉREZ DE LA CRUZ, M. Escolítidos (Coleóptera Scolytidae) asociados al agroecosistema cacao en Tabasco Mexico. XXXVIII(5). 2009

[30] CÓRDOVA, V. Factores que afectan la producción de cacao (Theobroma cacao L.). XXXIV(17). 2010

[31] IDICT, C. Cacao. EcuRed. 2015 
[32] RIVAS, M. Diversidad de Trichoderma spp. En plantaciones de Theobroma cacao L. Y su capacidad biocontroladora sobre Crinipellis perniciosa. XXXV(10). 2010

[33] OROZCO, C. Variabilidad genetica de Moniliophthora perniciosa en variedades de cacao (Theobroma cacao L.). LXI(2). 2012

[34] ENGELBRECHT, c. Ceratocystis Wilt of cacao-a disease of increasing importance Phytopathology. XCVII(12). 2009

[35] HANADA, R. Biocontrol potencial of trichoderma martiale against the black-pod disease (Phytophthora palmivora) of cacao. L(2). 2009

[36] CORPORACIÓN, Promoción de exportaciones e inversiones. (02 de Agosto de ). Corpei. Recuperado el 13 de Junio, de Corpei: http://ac.ciifenint.org/agroclima/index.php?option= com_content\&view=article\&id=49\&Itemid=77\#_ftn2 2010

[37] HERNÁNDEZ, A. Antagonistas microbianos para el manejo de la pudricion negra del fruto de Theobroma cacao L. XXIX(1). 2014

[38] ROMERO, X. Guía técnica del cultivo de cacao manejado con técnicas agroecológicas. San Salvador: Centro Cooperativo Sueco. 2011

[39] PANOZO, B. Efecto de biofungicidas orgánicos en el control de mazorca negra (Phytophthora palmivora, butl.) en cultivo de cacao. La Paz: Universidad Mayor De San Andres. 2009

[40] NEIL, E. Efecto de biofungicidas orgánicos en el control de mazorca negra (Phytophthora palmivora, butl.) en cultivo de cacao. La Paz: Universidad Mayor De San Andres. 2009

[41] PEREZ, M. Inpacto manejo y control de enfermedades causadas por Phytophthora. Pamplona: Universidad de Pamplona. 2010

[42] SÁNCHEZ, H. Determinación de la actividad enzimática extracelular de endo-1,3(4)- $\beta$-glucanasa y amplificación de genes endo-1,3(4)-glucanasa a partir de moniliophthora roreri. Tabasco: Colegio De Postgraduados. 2011

[43] ESTRELLA, E. Medidas de control de bajo impacto ambiental para mitigar la moniliasis (Moniliophthora roreri Cif y Par. Evans et al) en cacao híbrido nacional por trinitario. Santo Domingo: Escuela Politécnica Del Ejército. 2012

[44] TORRES DE LA CRUZ, M. Efecto de azoxystrobin sobre Moniliophthpra roreri, agente causal de la moniliasis del cacao (Theobroma cacao L.). XXXI(1). 2013

[45] PHILLIPS, W. Enfermedades del cacao en Centro America. I(93). 2009

[46] VILLAMIL, J. Evaluación in vitro de microorganismos nativos por su antagonismo contra Moniliophthora roreri Cif \& Par en cacao (Theobroma cacao L.). LXV(1). 2012

[47] SÁNCHEZ, F. Moniliophthora roreri (Cif y Par) Evans et al. en el cultivo de cacao. III(3). 2012
[48] MASTAHINICH, R. Estudio de factibilidad de la introduccion del clon CCN51. Zamorano: Zamorano. 2011

[49] SARABIA, W. Diagnóstico sobre la rehabilitacion y recuperación de la capacidad productiva de huertas tradicionales de cacao (Theobroma cacao L.). Milagro: Universidad Agraria Del Ecuador. 2008

[50] CÓRDOVA, V. Factores que afectan la producción de cacao (Theobroma cacao L.) en el ejido Francisco 1. Madero del Plan Chontalpa, Tabasco, Mexico. XVII(34). 2014

[51] FHIA. La moniliasis del cacao el enemigo a vencer. (20). 2012

[52] RAMÍREZ, S. El polisulfuro de calcio en el manejo de la moniliasis Moniliophthora roreri (Cif \& Par) Evans et al. del cacao Theobroma cacao L. XXIV(4). 2011

[53] VILLAVICENCIO, M. Caracterización morfológica, fisiológica y patogénica de Moniliophthora roreri aislados de cinco provincias de la costa Ecuatoriana. Guayaquil: Escuela Superior Politécnica Del Litoral. 2010

[54] VILLAMIL, J. Aplicación de Antagonistas Microbianos para el Control Biológico de Moniliophthora roreri Cif \& Par en Theobroma cacao L. Bajo Condiciones de Campo. LXVIII(1). 2015

[55] CORREA, J. Estado de la moniliasis de cacao causado por Moniliophthora roreri en colombia. LXIII(4). 2014

[56] SUÁREZ, L. Aislamiento microorganismos para control biológico de Moniliophthora roreri. LXII(4). 2013

[57] HERRERA, J. Use of Trichoderma fungi in spray solutions to reduce Moniliophthora roreri infection of Theobroma cacao fruits in Northeastern Costa Rica. LXII(3). 2014

[58] ASOCIADOS, J. Orius biotecnologia. (Disan Communications) Recuperado el 10 de junio, de Orius biotecnologia: www.oriusbiotecnologia.com. 2013

[59] CONSULCENTRO. Plan de desarrollo y ordenamiento territorial del cantón La Troncal. cuenca: Centro De Consultoria En Investigación, Planificación y Catastro. 2011

Recibido: 15 de abril de 2020

Aceptado: 3 de julio de 2020 